\title{
Trauma Craneoencefálico en el Departamento del Cesar, Colombia: Análisis de Frecuencia, Severidad y Complicaciones
}

\author{
Hernando Raphael Alvis-Miranda, Richard José Adie-Villafañe, Duffays Velasquez-Loperena, Robert Andres \\ Velasquez-Loperena, Zenen Carmona Meza, Gabriel Alcalá-Cerra, Luis Rafael Moscote-Salazar
}

\section{RESUMEN}

Objetivo: Analizar la epidemiología del traumatismo craneoencefálico durante los días de semana y los fines de semana en el departamento del Cesar, Colombia.

Materiales y métodos: Estudio retrospectivo, de base poblacional; se realizó análisis estadístico determinándose distribución de frecuencia a cada una de las variables de los datos obtenidos.

Resultados: Se analizaron las características epidemiológicas de los pacientes en el departamento del Cesar, la edad promedio de $38 \pm 18,8$, siendo $98(70,5 \%)$ del sexo masculino y $41(29,5 \%)$ del sexo femenino. De acuerdo al sexo en relación a las zona, la zona Norte $84(68,9 \%)$ hombres y $39(31,1 \%)$ mujeres, la zona centro 5 hombres $(100 \%)$ y la zona sur 9 hombres $(75 \%)$ y 3 mujeres $(25 \%)$, por otro lado se analizó la distribución de acuerdo al sexo y a la ocurrencia de traumas craneoencefálicos los fines de semana y días hábiles, el sexo masculino presentó 40 casos $(81,6 \%)$ y los días hábiles 72 casos $(69,2 \%)$, en relación al sexo femenino presento 9 casos $(18,4 \%)$ y los días hábiles $34(32,7 \%)$.

Conclusiones: El trauma craneoencefálico es un problema de salud pública. En el departamento del Cesar se requiere la implantación de estrategias de prevención de este tipo de lesiones. Los accidentes de tráfico continúan como primera causa, afectando, principalmente a la población joven masculina.

Palabras claves: Trauma craneoencefálico, Neuroepidemiología, Politrauma.

\section{ABSTRACT}

Objective: The purpose of this paper is to analyze the epidemiology of traumatic brain injury during week days and weekends in the department of Cesar, Colombia.

Materials and methods: A retrospective, population-based; it was performed statistical analysis for determining frequency distribution to each one of the variables.

Results: We analyzed the epidemiological characteristics of patients in the department of cesar, the average age of $38 \pm$ 18.8 , with $98(70.5 \%)$ males and $41(29.5 \%)$ females. According to sex in relation to the area, the North $84(68.9 \%)$ men and 39 $(31.1 \%)$ women, the central five men $(100 \%)$ and the South 9 men $(75 \%)$ and three women $(25 \%)$; a second distribution was analyzed according to sex and the occurrence of head trauma on weekends and working days, the male presented 40 cases $(81.6 \%)$ and 72 days cases $(69.2 \%)$, compared to females presented nine cases (18.4\%) and $34(32.7 \%)$ on week days.

Conclusion: Traumatic brain injury is a public health problem. In the department of Cesar is required the implementation of strategies for prevention of this type of injury. Traffic accidents remain the first cause, affecting mainly the young male population.

Keywords: Head trauma, Neuroepidemiology, Polytrauma.

How to cite this article: Alvis-Miranda HR, Adie-Villafañe RJ, Velasquez-Loperena D, Velasquez-Loperena RA, Meza ZC, Alcalá-Cerra G, Moscote-Salazar LR. Trauma Craneoencefálico en el Departamento del Cesar, Colombia: Análisis de Frecuencia, Severidad y Complicaciones. Panam J Trauma Critical Care Emerg Surg 2013;2(2):89-93.

Source of support: Nil

Conflict of interest: None declared

\section{INTRODUCCION}

El trauma craneoencefálico (TEC) se define como aquella lesión producto de un trauma contuso o penetrante o por fuerzas de aceleración/desaceleración asociados a uno o más de los siguientes: nivel de conciencia disminuido, amnesia, alteración neurológica o neuropsicológica objetivable, fractura de cráneo, diagnóstico de lesión intracraneal o lesión cefálica determinada como causa de muerte en certificado de defunción. ${ }^{1,2}$ Se constituye en la principal causa de mortalidad y morbilidad en la población infantil y en adultosjóvenes. ${ }^{3}$ En Estados Unidos, el TEC genera alrededor de 235.000 hospitalizaciones, 5000 muertes, y discapacidad en $99000,{ }^{3}$ y solo en ese país se estiman gastos de $\$ 110$ billones de dólares producto de costos directos (relacionados con la atención médica) e indirectos (relacionados a pérdida de productividad). ${ }^{4}$ El TEC, típicamente se clasifica según su severidad; tradicionalmente una puntuación en la Escala de Coma de Glasgow de 1415 es definida como trauma craneal leve, puntajes de 9-13 como moderada y puntajes menores de 8 como severo. El TCE leve es la forma más común, representando el $80-90 \%$ de todos los TCE. ${ }^{5}$

Saber cómo es el comportamiento cronológico del TEC nos permitiría ir un paso adelante en su manejo, conocer los picos temporales de su presentación y el mecanismo más frecuentemente involucrado, por ejemplo, nos ayudaría a encaminar de forma orientada los pasos de diagnóstico, tratamiento y rehabilitación de estos pacientes, especialmente cuando se conoce queColombia es un país en el que la causa principal de muerte es la violencia y quederivada de esta,un $49 \%$ y $70 \%$, son debidas a TEC. Según el DANE 
(Departamento Administrativo Nacional de EstadisticasColombia) ${ }^{6}$ en 1994 se presentaron en Colombia 40.046 muertes por traumas, siendo los homicidios responsables del 69,8\%, seguido por los accidentes de tránsito 15,9\%. El grupo etario más importante en cuanto a la mortalidad por trauma es el de los 14-34 años, en 2007 se reportaron 8053 muertes solo por trauma. ${ }^{6}$ El TEC al igual que otras enfermedades de evolución aguda puede ser susceptible de generar un peor pronóstico en función del momento de la semana en la que se produce, esto probablemente derivado de las condiciones en las que se ofrece el servicio de salud durante los fines de semana (e.g. menor personal sanitario), por ello se realizó el siguiente trabajo con el objetivo de evaluar las características de los pacientes con TEC ingresados a un centro de trauma en la ciudad de Valledupar, Cesar, al norte de Colombia durante los días de semana y los fines de semana.

\section{MATERIALES Y MÉTODOS}

\section{Diseño del Estudio}

Epidemiológico, observacional, retroprospectivo, de base poblacional.

\section{Ámbito}

Población de referencia de la clínica Laura Daniela de Valledupar, Cesar. El Cesar es uno de los 32 departamentos de Colombia, situado en la zona noreste del país, posee una extensión de $22.905 \mathrm{~km}^{2}$ y una población de 1.166 .420 habitantes.

\section{Recolección de Datos}

Se revisaron historias clínicas sistematizadas, desde enero de 2010 a enero de 2012, recogiéndose los siguientes datos: sexo, edad, fecha, hora y mecanismo del accidente, puntuación inicial en la Escala de Coma de Glasgow (GCS), factores de riesgo, tiempo de remisión, estancia en UCI, derivación a otro centro asistencial, fuga o muerte a la admisión) y destino final (alta domiciliaria, derivación a otro centro, fuga o muerte). De acuerdo a la puntuación en la GCS, se clasificó a los TCE en leves (14-15), moderados (9-13) y graves (3-8). Se discriminó la ocurrencia del TEC en los día de semana: de Lunes a Viernes, y los fines de semana: Sábado y Domingo.

\section{Análisis Estadístico}

Los datos fueron analizados a través del software 17.0 (SPSS. Inc., Chicago, IL). A los datos obtenidos se les realizó un análisis estadístico determinándosele distribución de frecuencia a cada una de las variables. Se consideró significativo un valor de $\mathrm{p}<0.05$. Se utilizo test de Fisher.

\section{RESULTADOS}

En total se reportaron 155 casos, de los cuales 139 correspondieron al departamento del Cesar, siendo 98 (70,5\%) del sexo masculino y 41 (29,5\%) del sexo femenino, con una edad promedio de $38 \pm 18,5$ años (Tabla 1 ).

\begin{tabular}{|c|c|}
\hline & $\begin{array}{c}\text { Casos reportados } \\
n=139\end{array}$ \\
\hline Edad en años ${ }^{a}$ & $38 \pm 18,8$ \\
\hline \multicolumn{2}{|l|}{ Sexo ${ }^{b, c}$} \\
\hline Hombre & $98(70,5)$ \\
\hline Mujer & $41(29,5)$ \\
\hline \multicolumn{2}{|l|}{ Mecanismo del trauma ${ }^{b, d}$} \\
\hline Accidente de tránsito & $114(82,0)$ \\
\hline Agresión física & $11(7,9)$ \\
\hline Caída & $13(9,3)$ \\
\hline Otros (Encontrado en vía pública) & $1(0,7)$ \\
\hline \multicolumn{2}{|l|}{ Frecuencia durante de la semana ${ }^{b}$} \\
\hline Día de semana & $96(69,1)$ \\
\hline Fines de semana & $43(30,9)$ \\
\hline
\end{tabular}

${ }^{a}$ Media con desviación estándar. ${ }^{b}$ Frecuencia absoluta (Porcentaje). 'Valor $p=0,29 .{ }^{d}$ Valor $p=0,04$

De acuerdo al sexo en relación a la zona, se halló que en la zona norte hubo $84(68,9 \%)$ hombres y $39(31,1 \%)$ mujeres, en la zona centro 5 hombres $(100 \%)$ y en la zona sur 9 hombres (75\%) y 3 mujeres (25\%).

La distribución de acuerdo al sexo y a la ocurrencia de TEC [fines de semana y durante los días hábiles (de lunes a viernes)] se encontró, que el sexo masculino presentó 34 casos $(34,7 \%)$ durante el fin de semana y en los días hábiles 64 casos $(65,3 \%)$, en el grupo de sexo femenino hubo 9 casos $(21,9 \%)$ en fines de semana y durante los días hábiles $32(78,1 \%)$.

Dentro del total de casos del departamento de Cesar, en 114 casos $(82,0 \%)$ el TEC fue secundario a accidente de tránsito, 13 casos $(9,3 \%)$ fueron debido a caídas, 11 casos $(7,9 \%)$ a agresión física y el resto un $0,7 \%$, su mecanismo de trauma no fue concluyente debido a que el paciente fue hallado en la vía pública (Tabla 1).

La ECG promedio fue de 13/15 $\pm 3,09$, con una severidad del TEC en 93 casos (66,9\%) siendo leve, en 31 casos $(22,3 \%)$ siendo moderado. La estancia en UCI fue requerida en 53 casos $(38,1 \%)$ con una estancia promedio por paciente de $8,75 \pm 8$ días, siendo el tiempo de remisión de $13,1 \pm 39$ horas. $34(24,4 \%)$ pacientes presentaron complicaciones. De los pacientes que requirieron manejo quirúrgico, 15 casos $(30,6 \%)$ se les hizo craneotomía y 4 casos $(8,2 \%)$ fueron manejados con craniectomía descompresiva (Tabla 2). 
Trauma Craneoencefálico en el Departamento del Cesar, Colombia: Análisis de Frecuencia, Severidad y Complicaciones

Tabla 2: Características clínicas de los pacientes

\begin{tabular}{|c|c|}
\hline & $\begin{array}{c}\text { Casos reportados } \\
n=139\end{array}$ \\
\hline Glasgow ingreso $(15 / 15)^{a}$ & $13 / 15 \pm 3,09$ \\
\hline \multicolumn{2}{|l|}{ Severidad del TEC ${ }^{b}$} \\
\hline Leve & $93(66,9)$ \\
\hline Moderado & $31(22,3)$ \\
\hline Severo & $15(10,8)$ \\
\hline \multicolumn{2}{|l|}{ Estancia en $\mathrm{UCl}$} \\
\hline Requerimiento $^{\mathrm{b}}$ & $53(38,1)$ \\
\hline Tiempo en días ${ }^{a}$ & $8,75 \pm 8$ \\
\hline \multicolumn{2}{|l|}{ Manejo del TEC ${ }^{b}$} \\
\hline Manejo Conservador & $91(65,5)$ \\
\hline Manejo Quirúrgico & $48(34,5)$ \\
\hline Craneotomía & $15(30,6)$ \\
\hline Craniectomía & $4(8,2)$ \\
\hline Otros procedimiento & $29(59,2)$ \\
\hline Tiempo de remisión en horas ${ }^{a}$ & $13,1 \pm 39$ \\
\hline Complicaciones $^{b}$ & $34(24,4)$ \\
\hline
\end{tabular}

${ }^{a}$ Media más desviación estándar. ${ }^{b}$ Valor absoluto (Porcentaje)

En relación al mecanismo de trauma y la necesidad de manejo quirúrgico, se halló que 45 casos $(39,5 \%)$ de pacientes con accidente de tránsito requirieron cirugía, y 2 casos $(18,1 \%)$ de TEC secundario a agresión física la requirieron.

En los fines de semana la ocurrencia de TEC fue de 96 casos $(69,1 \%)$ del total de la muestra y 43 de los casos (30,9\%) se dieron durante los días hábiles (Tabla 1).

Tanto los días hábiles como los fines de semana, en la mayoría de los casos la severidad del TEC fue leve, reportándose respectivamente 60 casos $(62,5 \%)$ y 33 casos (76,6\%). El mecanismo del trauma más frecuente los día hábiles fueel accidente de tránsito con 83 casos $(86,5 \%)$, seguido de las caídas con 8 casos ( $8,3 \%)$; los fines de semana el mecanismo más frecuente también fue el accidente de tránsito con $31(72,1 \%)$ casos, seguido de las agresiones físicas con $6(13,9 \%)$ (Tabla 2).

De acuerdo a la ocurrencia de trauma aislado y politrauma se encuentra que tanto los fines de semana como los días hábiles, se presenta en mayor frecuencia el trauma aislado con 25 casos $(58,1 \%)$ y 56 casos $(58,3 \%)$ respectivamente (Tabla 3).

De la severidad TEC de acuerdo a la zona en el departamento del Cesar se reportó, que en la zona norte se presentaron $88(72,1 \%)$ traumas leves, $23(18,9 \%)$ traumas moderados y $11(9,0 \%)$ traumas severos, en la zona centro 2 traumas leves(40\%), traumas moderados $2(40 \%)$ y traumas severos $1(20 \%)$, en la zona sur se presentaron $3(25 \%)$ traumas leves, $6(50)$ traumas moderados y $3(25 \%)$ traumas severos.
Tabla 3: Características del TEC durante los días de semanas y los fines de semana en el departamento de Cesar

\begin{tabular}{|c|r|r|r|} 
& \multicolumn{3}{|c|}{$\begin{array}{c}\text { Casos reportados } \\
n=139\end{array}$} \\
\hline & Día hábil & $\begin{array}{c}\text { Fin de } \\
\text { semana }\end{array}$ & Valor $p$ \\
\hline Severidad del TEC & & \\
\hline Leve & $60(62,5)$ & $33(76,7)$ & 0,06 \\
\hline Moderado & $23(24,0)$ & $8(18,6)$ & \\
\hline Severo & $13(13,5)$ & $2(4,6)$ & \\
\hline Mecanismo del Trauma & & & 0,05 \\
\hline Accidente de Transito & $83(86,5)$ & $31(72,1)$ & \\
\hline Agresión Física & $5(5,2)$ & $6(13,9)$ & \\
\hline Caídas & $8(8,3)$ & $5(11,6)$ & \\
\hline Otros & - & $1(2,3)$ & \\
\hline Tipo de Trauma & & & 0,15 \\
\hline Aislado & $56(58,3)$ & $25(58,1)$ & \\
\hline Politrauma & $40(41,7)$ & $18(41,9)$ & \\
\hline
\end{tabular}

avalor absoluto (Porcentaje)

En la zona norte, $101(82,8 \%)$ casos fueron debido a accidentes de tránsito, $9(7,4 \%)$ a agresiones físicas, $11(9,0 \%)$ por caídas y otros $1(0,8 \%)$; en la zona centro $4(80,0 \%)$ casos fueron por accidente de tránsito y $1(20 \%)$ caso por caída y en la zona sur, 9 (75\%) casos por accidente de tránsito, $2(16,7 \%)$ por agresión física y $1(8,3 \%)$ por caída.

Se analizó la presencia de complicaciones de acuerdo a la ocurrencia del TEC los fines de semana o días hábiles, encontrándose que los fines de semana, se presentaron $11(22,4 \%)$ complicaciones y los días hábiles 29 (27,9\%).

En cuanto a la frecuencia de los TEC en cada zona y los meses, se evidenció que durante el mes de abril hubo un primer pico en la zona norte con $13(10,7 \%)$ caso y un segundo pico en los meses de septiembre con $17(13,9 \%)$, octubre con $26(21,3 \%)$ y noviembre con $13(10,7 \%)$. En la zona centro se evidenciaron casos esporádicos entre los meses de febrero, 1 (20\%), marzo, 2 (40\%) y abril, $1(20 \%)$ y en la zona sur dos periodos de casos, el primero entre los meses de febrero, 1 (8,3\%) caso, en marzo, abril y mayo se presentaron $2(16,7 \%)$ casos por mes y en junio 3 (25\%) casos; el segundo periodo en los meses de octubrey noviembre con $1(8,3 \%)$ caso cada uno.

\section{DISCUSIÓN}

El TEC o lesión traumática cerebral tiene un amplio rango de consecuencias deletéreas, que representa al menos la mitad de las muertes por trauma, hecho que impacta a los sistemas de salud por los elevados costos de su manejo incluyendo la rehabilitación de los pacientes. ${ }^{7-9}$ El TEC es un problema importante en todo el mundo. ${ }^{10-13}$

Nuestros resultados evidencian un comportamiento similar al reportado en la literatura respecto a edad y 
mecanismo de trauma, evidenciamos una edad promedio de $38 \pm 18,8$ años y como principal mecanismo causal de TEC a los accidentes automotores. Se conoce que en general, individuos de 0-4 años, 15-24 años, y de 75 años en adelante se encuentran en alto riesgo de sufrir TEC, siendo las principales causas los accidentes automotores, las caídas y la violencia. ${ }^{14}$ La evidencia disponible reporta una mortalidad por TEC entre el $35-42 \%$, especialmente en pacientes con edades entre 15-25 años. ${ }^{8,12,14}$ Además, en nuestro trabajo los TEC fueron más frecuentes en el sexo masculino.

En nuestro trabajo decidimos dividir para conceptos prácticos al departamento del Cesar en tres zonas (norte, centro y sur), la zona norte teniendo como correspondencia a la ciudad de Valledupar y las zonas aledañas, la zona centro teniendo como referencia al municipio de Chiriguaná y la zona sur al municipio de Aguachica. En la zona norte fueron más frecuentes los politraumas/TEC, probablemente en relación a que esta zona comprende la capital del departamento (Valledupar). Los TEC fueron más frecuentes en la zona urbana.

Distintos análisis del comportamiento del TEC a los largo del período determinado como semana, que consta de los días hábiles (lunes a viernes) y fin de semana (sábado y domingo), han permitido evidenciar la existencia de un fenómeno que se ha denominado 'efecto fin de semana' (Weekendeffect) en el que las admisiones hospitalarias en estos días de la semana se asocian con mayor mortalidad, se ha demostrado que la mortalidad de los pacientes ingresados durante el fin de semana es superior a la de quienes ingresan durante los días laborables o hábiles, ${ }^{15}$ especialmente los ingresados por emergencias cardiovasculares, enfermedad cerebrovascular aguda, pero en el trauma en general no ha sido bien definido, sin embargo existen algunos estudios que evalúan tal relación.

En este estudio se observó una mayor severidad del TEC durante los días hábiles en comparación con los ingresos los fines de semana, en este último el TEC fue predominantemente leve $(76,6 \% ; p=0,6$; Tabla 3$)$, el resto de variables se comportó de manera similar en ambos momentos de la semana. Tales hallazgos guardan relación con lo reportado en la literatura.

En 2010, Carr et al, ${ }^{16}$ estimaron los riesgos relativos de mortalidad asociada a los ingresos en los fines de semana y en horas nocturnas. Deun total de 4382 pacientes, de los cuales el $34 \%$ fue ingresado en fines de semana y $23,3 \%$ lo hicieron en horas de la noche (12:00 medianoche a 6:00 am), determinaron queen general el escore de severidad de la lesión (InjurySeverity Score) fue de 13,7 para ambos momentos de la semana y del día. La ECG promedio fue de 13.6, sin disparidades en tales momentos. En los análisis ajustados para controlar edad, sexo, el escore de severidad de la lesión, la ECG y la hipotensión al ingreso no se observó diferencias entre los ingresados en los fines de semana (OR, 1,03, IC 95\%, 0,71-1,51) y los ingresados en horario nocturno (OR, 0,79, IC 95\%, 0,49-1,25). Es apreciable que no hay mortalidad diferencial, sin embargo debe tenerse en cuenta que hay variables que son totalmente independientes a la hora del día o el día de semana que tienen requerimientos explícitos de atención en centros de trauma.

En 2012, Schneider et al ${ }^{17}$ determinaron la mortalidad porTEC severo, aunque solamente en pacientes adultos mayores admitidos los fines de semana en comparación a los admitidos en días hábiles. De un total de 38675 pacientes, el25.6\% fue admitido en fines de semana, siendo las mujeres las principalmente ingresadas (51.6 vs 50.2\%, $\mathrm{p}=0.022)$.Encontraron que los pacientes admitidos los fines de semana tenían un ligero menor porcentaje de comorbilidades $(p<0.001)$ y de menor severidad del TEC $(p<0.001)$. La estancia hospitalaria en los ingresados los fines de semana fue menor (4 vs 5 días, $\mathrm{p}<0,001$ ), sin embargo, los gastos económicos no difirieron $(\$ 27,128$ vs $\$ 27,703, p=0.667)$;pero la mortalidad proporcional fue mayor en los pacientes del grupo fin de semana $(9,3 \% v s$ $8,4 \%, \mathrm{p}=0,008)$, y se demostró un incremento del $14 \%$ en el riesgo de mortalidad.

A modo de conclusión de este estudio, los pacientes adultos mayores admitidos en fines de semana son menos severamente lesionados, acarrean menos comorbilidad y generan gastos totales similares en comparación a aquellos admitidos en los restantes días de la semana, pero, como fue demostrado hay un mayor riesgo de mortalidad, por tanto deben determinarse y eliminarse los mecanismos causales de tal disparidad.

Algunas explicaciones se han brindado para este fenómeno, entre las que se encuentran un número reducido de personas trabajando en fines de semana en los hospitales, lo cual puede condicionar una reducción en los cuidados de los pacientes durante esos días. Los procesos tiempo sensibles (por ejemplo, infarto de miocardio con elevación del ST, la ECV aguda y el paro cardiaco) generan implicaciones respecto al personal que los atiende y a los sistemas de solución de los mismos, por lo tanto para estos procesos deben desarrollarse sistemas de categorización basados en las capacidades del cuidado en los servicios de urgencias, de requerimientos específicos respecto al personal de atención médica y se requiere además de un programa de mejora de calidad en los sistemas de emergencias para suplir las consecuencias del efecto fin de semana.

Desafortunadamente el diseño metodológico no permite realizar conclusiones de gran peso estadístico, por su naturaleza principalmente retrospectiva, y la probabilidad de sesgos, especialmente el hecho de que en las zonas en 
la periferia del departamento, los pacientes pueden ser remitidos a centros de otros departamentos con capitales más cercanas respecto a Valledupar, la ciudad capital del Cesar.

\section{CONCLUSIONES}

A la vista de los resultados de este trabajo se puede definir un patrón de pacientes comprometidos con TEC en el departamento del Cesar, Colombia. Usualmente pacientes jóvenes, provenientes de la zona norte del departamento, se ven implicados con un trauma de alta energía, con severo deterioro neurológico en los meses de abril, mayo, septiembre, octubre y noviembre. Requiriendo manejo en unidad de terapia intensiva, y manejo neuroquirúrgico en las primeras 24 horas. Por lo que se requiere de la implantación de medidas de prevención de traumas craneoencefálicos en el departamento del Cesar, Colombia.

\section{REFERENCIAS}

1. Coronado VG, McGuire LC, Sarmiento K, Bell J, Lionbarger MR, Jones CD, Geller AI, Khoury N, Xu L. Trends in traumatic brain injury in the US and the public health response: 1995-2009. J Safety Res 2012;43:299-307.

2. Centers for Disease Control and Prevention. Traumatic brain injury in the united states: a report to congress. 1999 p. 20-40.

3. Goodman MD, Makley AT, Lentsch AB, Barnes SL, Dorlac GR, Dorlac WC, Johannigman JA, Pritts TA. Traumatic brain injury and aeromedical evacuation: when is the brain fit to fly? J Surg Res 2010;164:286-293.

4. Graves JM, Sears JM, Vavilala MS, Rivara FP. The burden of traumatic brain injury among adolescent and young adult workers in Washington State. J Safety Res 2013 Jun;45:133-139.

5. Bigler ED, Maxwell WL. Understanding mild traumatic brain injury: neuropathology and neuroimaging. In: Vasterling J, Bryant R, Keane T, editors. PTSD and Mild Traumatic Brain Injury, New York: Guilford Press 2012. p. 60-65.

6. DANE. Defunciones no fetales. Defunciones No Fetales. p. 10-20.

7. Choi BY, Jang BG, Kim JH, Lee BE, Sohn M, Song HK, Suh SW. Prevention of traumatic brain injury-induced neuronal death by inhibition of NADPH oxidase activation. Brain Res 2012;1481:49-58.

8. Engel DC, Mikocka-Walus A, Cameron PA, Maegele M. Prehospital and in-hospital parameters and outcomes in patients with traumatic brain injury: a comparison between German and Australian trauma registries. Injury 2010;41:901-906.

9. Arango-Lasprilla JC, Ketchum JM, Cifu D, Hammond F, Castillo C, Nicholls E, Watanabe T, Lequerica A, Deng X. Predictors of extended rehabilitation length of stay after traumatic brain injury. Arch Phys Med Rehabil 2010;91:1495-1504.

10. Yuan Q, Liu H, Wu X, Sun Y, Yao H, Zhou L, Hu J. Characteristics of acute treatment costs of traumatic brain injury in Eastern China--a multi-centre prospective observational study. Injury 2012;43:2094-2099.

11. Pérez K, Novoa AM, Santamariña-Rubio E, Narvaez Y, Arrufat V, Borrell C, Cabeza E, Cirera E, Ferrando J, García-Altés A, et al. Incidence trends of traumatic spinal cord injury and traumatic brain injury in Spain, 2000-2009. Accid Anal Prev 2012;46: 37-44.

12. Arabi YM, Haddad S, Tamim HM, Al-Dawood A, Al-Qahtani S, Ferayan A, Al-Abdulmughni I, Al-Oweis J, Rugaan A. Mortality reduction after implementing a clinical practice guidelines-based management protocol for severe traumatic brain injury. J Crit Care 2010;25:190-195.

13. Kan EM, Ling E-A, Lu J. Microenvironment changes in mild traumatic brain injury. Brain Research Bulletin 2012;87: 359-372.

14. Engberg A. Severe traumatic brain injury--epidemiology, external causes, prevention, and rehabilitation of mental and physical sequelae. Acta Neurol Scand Suppl 1995;164:1-151.

15. Bell CM, Redelmeier DA. Mortality among patients admitted to hospitals on weekends as compared with weekdays. N Engl J Med 2001;345:663-668.

16. Carr BG, Jenkins P, Branas CC, Wiebe DJ, Kim P, Schwab CW, Reilly PM. Does the trauma system protect against the weekend effect? J Trauma 2010;69:1042-1047; discussion 1047-1048.

17. Schneider EB, Hirani SA, Hambridge HL, Haut ER, Carlini AR, Castillo RC, Efron DT, Haider AH. Beating the weekend trend: increased mortality in older adult traumatic brain injury (TBI) patients admitted on weekends. J Surg Res 2012;177:295-300.

\section{ACERCA DE LOS AUTORES}

\section{Hernando Raphael Alvis-Miranda}

Medico, Universidad de Cartagena

\section{Richard José Adie-Villafañe}

Medico, Universidad de Cartagena

\section{Duffays Velasquez-Loperena}

Medico, Universidad del Magdalena

\section{Robert Andres Velasquez-Loperena}

Medico, Universidad del Magdalena

\section{Zenen Carmona Meza}

Epidemiólogo, Universidad de Cartagena

\section{Gabriel Alcalá-Cerra}

Residente de Neurocirugía, Universidad de Cartagena, Grupo de Investigación en Ciencias de la Salud y Neurociencias (CISNEURO)

\section{Luis Rafael Moscote-Salazar}

Neurocirujano, Universidad de Cartagena, Grupo de Investigación en Ciencias de la Salud y Neurociencias (CISNEURO)

Correspondencia: Luis Rafael Moscote-Salazar, Universidad de Cartagena, Cartagena de Indias. Grupo de Investigación en Ciencias de la Salud y Neurociencias (CISNEURO), Colombia, e-mail: mineurocirujano@aol.com/cisneuro.investigacion@gmail.com 